\title{
Using self-reliance factors to decide how to share control between human powered wheelchair drivers and ultrasonic sensors
}

\author{
David A Sanders TD VR
}

\begin{abstract}
A shared-control scheme for a powered wheelchair is presented. The wheelchair can be operated by a wheelchair driver using a joystick, or directed by a sensor system, or control can be combined between them. The wheelchair system can modify direction depending on the local environment. Sharing the control allows a disabled wheelchair driver to drive safely and efficiently. The controller automatically establishes the control gains for the sensor system and the human driver by calculating a self-reliance factor for the wheelchair driver. The sensor system can influence the motion of the wheelchair to compensate for some deficiency in a disabled driver. Practical tests validate the proposed techniques and designs.
\end{abstract}

Index Terms-Wheelchair; Disabled; Driver; Self-reliance; Shared-control.

\section{INTRODUCTION}

$\mathrm{O}$ LDER or disabled people can lack the strength or ability needed to self-propel a manual wheelchair but a considerable amount of cognitive skill is needed to safely operate a powered wheelchair [1]. Potential users with cognitive impairments tend to be excluded from powered wheelchair driving [2]. That lack of mobility can lead to undesirable mental, social, and physical effects [3, 4].

Robots can perform some tasks without any human involvement [5] but in many situations, a wheelchair driver can benefit from intervention by the sorts of sensor system used by mobile robots [6]. Wheelchair control has been extensively studied [7-11]. Despite that, powered wheelchair control is still challenging because the driver is disabled [12], the environment is largely unknown and complicated, and it is often difficult to understand what (and how much) assistance is really needed [13]. Devices to assist users are sometimes necessary [14].

An assortment of unpredicted situations may occur [15]. They can affect a wheelchair driver and wheelchair operation [16]. With some collaboration with a sensor system [17], a wheelchair driver may handle complicated tasks in a more efficient manner [18].

This paragraph of the first footnote will contain the date on which you submitted your paper for review.

This work was supported in part by The Royal Academy of Engineering and the University of Portsmouth.

D. A. Sanders is a Reader at the University of Portsmouth, UK (e-mail: david.sanders@port.ac.uk).
If a sensor system is introduced then an interface is required to share control between a powered wheelchair driver and the sensor system. Kuniaki et. al. [19] described a collaborative tele-operation system that allowed users to remotely determine behaviour and implement changes in real time. Macharet et. al. [20] described a tele-presence system that allowed a user to send a target position and conduct tele-operation. In the work described in this paper, an ultrasonic transmitter sends a burst every second so that the powered wheelchair can detect the direction of a target by simple triangulation.

The system allows intimate collaboration between a sensor system and a wheelchair driver by combining commands from the human driver and the wheelchair sensor system. In [21], a two-tiered, combined-control architecture was described that promoted cooperation between a robotic system and a human director. In [22], a new combined-control system was presented that allowed an operator to obtain feedback from automated processes in order to improve performance and reduce workload. To improve safety and reduce workload for vehicle systems [23], [24], autonomous systems can assist a driver. Satti et. al. [25] proposed combined-control using a brain-computer interface. In [26] a controller is described for controlling groups of quadcopters with collision-avoidance and formation-control.

Cooperative interaction between a human driver and a powered wheelchair can be categorized as: assisting with behaviour, haptic feedback and blending commands. In assisting with behaviour approaches, a user's command is constrained, although the wheelchair may not generate new commands [9]. Haptic combined-control allows a human to provide commands directly to a wheelchair using a hapticdevice [27]. In blending commands, a human user and a system provide commands simultaneously and they are fused with specific ratios [28]. The distribution of the authority is important for human driver / powered wheelchair cooperation. Various interfaces can be used [29-35]

In [36], Carlson et. al. proposed a method to predict a user's proposed direction and correct the control signals to accomplish a desired direction of travel. Optimized parameters were acquired from experiments [37], which worked in specific cases and dynamic-distribution establishes a function to adjust any distribution in real-time [38]. For example, in [39] weights were adjusted by evaluating user commands. 
This paper introduces new methods to assist a powered wheelchair driver in complex and changing environments. By combining human driver commands and suggested behaviour from a sensor system, both a disabled driver and sensor system cooperate to achieve safe motion. A method is created that permits a sensor system to know the status of a wheelchair and then to command the wheelchair.

The direction of the wheelchair is controlled so that it turns to face a desired direction. The wheelchair then maintains the direction while avoiding obstacles. At times, it is easier and more efficient for the sensor system to control the wheelchair. However, there were not any studies of variable shared-control of powered wheelchairs in the literature.

The structure of this paper is as follows. The powered wheelchair and sensor systems are described in Section II, including the behaviour of the wheelchair as that is important for shared-control. Controller design is presented in III and the new shared controller is described in IV. Some experimental results are included in $\mathrm{V}$ and the contribution of the work is summed up in VI. Some considerations for future work are included in VII.

\section{THE POWERED WHEELCHAIR AND SENSOR SYSTEMS}

A Bobcat II Wheelchair was used in this research $[9,13]$.

The system consisted of: the mobile powered wheelchair, an ultrasonic sensor system for obstacle avoidance, the new shared controller and inputs for the driver and a sensor system. Ultrasonic range finders on the front of the wheelchair detect obstacles. An on-board computer processes sensory data and adjusts the direction and speed of the wheelchair.

\section{A. The powered wheelchair}

Steer-and-drive was used for the front two driving wheels of the four-wheeled powered wheelchair. Each driving wheel has a motor that drives independently so that the wheelchair is steered by varying the drive to each motor. The steer-anddrive wheelchair can turn on a center of rotation [40]. If $\mathrm{V}$ is the linear velocity of the wheelchair, $\phi$ is direction and $\omega$ is the angular velocity for the powered wheelchair, then the velocity at the center of mass for the wheelchair platform base is

$$
\boldsymbol{V}_{\text {center }}=(\mathrm{V}, \phi, \omega)
$$

The steering angle and wheel speeds are produced and sent to the wheel servo-controllers. As the wheelchair moves, odometry calculates the rough location of the wheelchair using shaft encoders and a simple model of obstacles that the wheelchair has detected can be built in a simple SLAM Unit. The kinematic model used is described in [41] and the type of odometer design is that described in [42].

The powered wheelchair had two driving-wheels on the same axis. Motion and orientation were achieved by driving the wheels independently.

\section{B. Ultrasonic sensor system}

Simultaneous localization and mapping is useful for wheelchair navigation and a SLAM algorithm was developed for the powered wheelchair. Fig. 1 shows the beam patterns of two sensors.

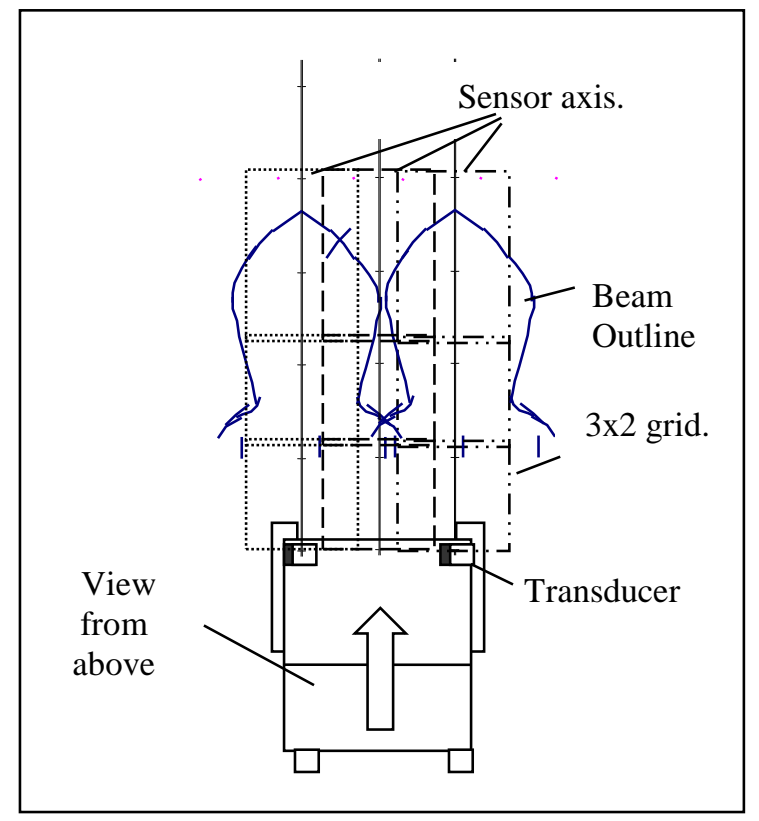

Fig. 1. Array created by overlapping beam patterns of two ultrasonic sensors.

Ultrasonic range scans provided information about obstacles ahead of the wheelchair. The position of obstacles was recorded as polar coordinates. The odometers recorded the path of the wheelchair. Point to point matching between the odometer inputs and the sensor inputs allowed the position of obstacles to be recorded in a simple database as a translation and a rotation.

In each ultrasonic range scan, the data were described as $\left(\mathrm{x}, y, \theta\{\mathrm{r}, \phi\}^{\left.\mathrm{n}_{\mathrm{i}=1}\right)}\right.$, where $\mathrm{x}, y, \theta$ are the current pose, $\mathrm{r}$ is the range and $\phi$ is the bearing of the scan.

Scan data in polar coordinates are:

$$
\begin{aligned}
& \left.r_{\text {current }}=\sqrt[V]{ }\left\{\left(r_{s c a n} \cos \theta_{\mathrm{t}}+\theta_{\mathrm{t}}\right)^{2}+x_{\mathrm{t}}{ }^{2}\right)+\left(r \cdot \sin \left(\theta_{\mathrm{t}}+\theta_{\mathrm{t}}\right)^{2} y_{\mathrm{t}}{ }^{2}\right)^{2}\right\} \\
& \left.\left.\phi^{\prime}{ }_{\mathrm{pi}}=\operatorname{atan} 2\left\{\left(r_{s c a n} \sin \theta_{\mathrm{t}}+\phi\right)+x_{\mathrm{t}}{ }^{2}\right)+y_{\mathrm{t}} r_{s c a n} \cos \left(\theta_{\mathrm{t}}+\phi\right)+x_{\mathrm{t}}\right)\right\}
\end{aligned}
$$

Current range, $r_{\text {current }}$, can be aligned to reference scan range, $\mathrm{r}_{\text {scan }}$ to determine wheelchair position $\left(x_{\mathrm{r}}, y r\right)$, and minimize error between the current scan data, $\mathrm{r}_{\text {current }}$, and reference scan,

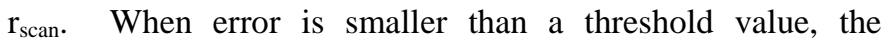
estimation is considered to have converged. To estimate rotation, the current scan was adjusted to match the reference scan to determine the wheelchair's orientation, $\theta_{\mathrm{t}}$, which minimizes error between $r_{\text {current }}$ and $r_{\text {scan }}$. Translation and rotation estimates were calculated by iteration.

Because noise errors may exist in the odometer system, the method was not adequate for localization over long distances. An extended Kalman filter algorithm combined the odometry 
and the sensor result so that localization error for the powered wheelchair was reduced.

In the extended Kalman filter algorithm, the land-mark scan is defined as: $\boldsymbol{\mu}_{\mathrm{t}, \text { new }}=\left[\boldsymbol{x}_{\mathrm{v}} . . \boldsymbol{y}_{1} . ., \boldsymbol{y}_{\mathrm{i}}\right]$ where $\boldsymbol{x}_{\mathrm{v}}$ is the wheelchair's pose, $\boldsymbol{y}_{\mathrm{n}}=\left[x_{n}, y_{\mathrm{n}}, \theta_{\mathrm{n}}\right]$ is the $\mathrm{n}^{\text {th }}$ landmark and $\mathrm{n}=1$ to $\mathrm{i}$, where $\mathrm{i}$ is the newest landmark. The wheelchair motion speed is defined as $\mathbf{u}_{\mathrm{t}}=\left[V_{x t}, V_{\mathrm{yt}}, \omega_{\mathrm{t}}\right]^{\mathrm{T}}$ and the wheelchair 's state is expressed as:

$$
\mathrm{q}_{t}=\left[\begin{array}{l}
x_{t} \\
y_{t} \\
\theta_{t}
\end{array}\right]=\left[\begin{array}{l}
x_{t-1} \\
y_{t-1} \\
\theta_{t-1}
\end{array}\right]+\left[\begin{array}{c}
A \\
B \\
\omega_{t}
\end{array}\right]
$$

Where:

$A=V_{\mathrm{xt}} \Delta_{\mathrm{t}} \sin \left(\theta_{\mathrm{t}-1}+\omega_{\mathrm{t}} \Delta_{\mathrm{t}} / 2\right)+V_{\mathrm{yt}} \Delta_{\mathrm{t}} \cos \left(\theta_{\mathrm{t}-1}+\omega_{\mathrm{t}} \Delta_{\mathrm{t}} / 2\right)$

and

$B=V_{\mathrm{xt}} \Delta_{\mathrm{t}} \cos \left(\theta_{\mathrm{t}-1}+\omega_{\mathrm{t}} \Delta_{\mathrm{t}} / 2\right)+V_{\mathrm{yt}} \Delta_{\mathrm{t}} \sin \left(\theta_{\mathrm{t}-1}+\omega_{\mathrm{t}} \Delta_{\mathrm{t}} / 2\right)$

Where $\Delta_{\mathrm{t}}$ is the sampling time, $V_{\mathrm{xt}}$ and $V_{\mathrm{yt}}$ are the measured linear velocity of the wheelchair in the $\mathrm{x}$ and $\mathrm{y}$ direction and $\omega_{t}$ is the measured angular velocity of the wheelchair.

The ultrasonic scan and the odometers were used to build a simple map in a database. The system vector and information from measurements were used to update system state and covariance.

\section{CONTROL}

A goal-seeking controller allowed the wheelchair to move and follow commands from a disabled driver and an automatic obstacle-avoider ensured the wheelchair did not collide with anything.

\section{A. Controller}

The linear velocity of the wheelchair and the angular velocity were considered. The wheelchair could track a desired direction at a desired linear velocity when the heading of the wheelchair was at an arbitrary angle, as shown in Fig. 2. The control laws for wheelchair linear velocity and angular velocity are shown here. To track the target position, the control law for the wheelchair's linear velocity, $V_{\mathrm{r}}$ was:

$V_{\mathrm{r}}=\mathrm{V}_{\mathrm{Max}} \mathrm{X} \mathrm{D} / \mathrm{D}_{\text {DesSp }}$ when $|\mathrm{D}|<\mathrm{D}_{\text {DesSp }}$

and

$V_{\mathrm{r}}=\mathrm{V}_{\operatorname{Max}} \mathrm{x} \mathrm{D} / \mathrm{D}_{\text {DesSp }}$ when $|\mathrm{D}|>\mathrm{D}_{\text {DesSp }}$

where $\mathrm{D}$ is the vector from the wheelchair joystick, $V_{\mathrm{Max}}$ is the maximum speed of the wheelchair and $\mathrm{D}_{\text {DesSp }}$ is the demand speed. If the vector from the wheelchair joystick is greater than the sensor range, the wheelchair moves at the desired speed.

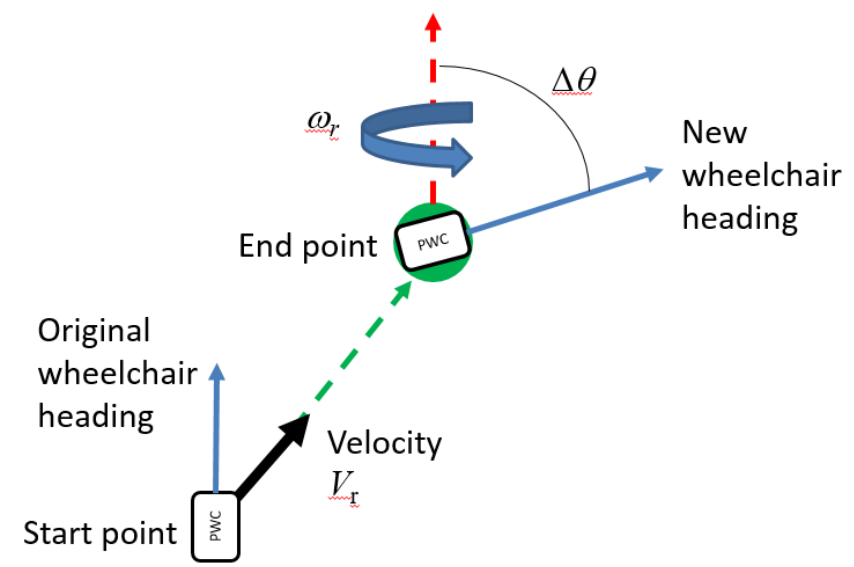

Fig. 2. Controller design.

The wheelchair's heading changes during traveling. To track the wheelchair's heading, the control law for the wheelchair's angular velocity, $\omega_{r}$, is defined as:

$\omega_{r}=\omega_{\text {Max }} \mathrm{x} \Delta \theta / \theta_{\text {DesHd, }}$ when $|\Delta \theta|<\theta_{\text {DesHd }}$

and

$\omega_{r}=\omega_{\operatorname{Max}} \mathrm{X} \Delta \theta /|\Delta \theta|, \quad$ when $|\Delta \theta|>\theta$ DesHd

Where $\omega_{\text {Max }}$ is the maximum value for the wheelchair's angular velocity and $\theta_{\text {DesHd }}$ is the desired heading, and the current heading of the wheelchair is expressed as the wheelchair's heading error, $\Delta \theta$. If the wheelchair's heading error is greater than the buffer angle, the wheelchair turns. If the wheelchair's heading error is less than the buffer angle, the control law adjusts the angular velocity and the track to the desired heading.

\section{B. Obstacle avoider}

Obstacle-avoidance ensured the wheelchair did not collide with anything during motion. The obstacle-avoider took advantage of the omnidirectional mobility of the wheelchair. The speed and direction of the wheelchair could be denoted by a vector quantity. When a wheelchair moved near to an obstacle, the potential field method was used so that the avoider generated a repulsive force and the wheelchair moved away from the obstacle, as shown in Fig. 3.

The avoidance velocity, $\mathbf{V}_{\mathrm{o}}$ can be expressed as:

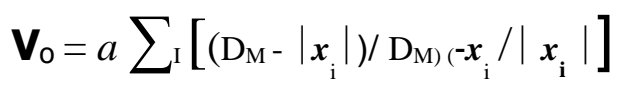

where $\boldsymbol{x}_{\mathrm{i}}$ are vectors for obstacle positions ahead of the wheelchair, $\mathrm{D}_{\mathrm{M}}$ was a safe distance and $a$ is a constant. Positions of obstacles, $x_{i}$, were detected by ultrasonic sensors and odometer information about position was recorded in a simple database. 


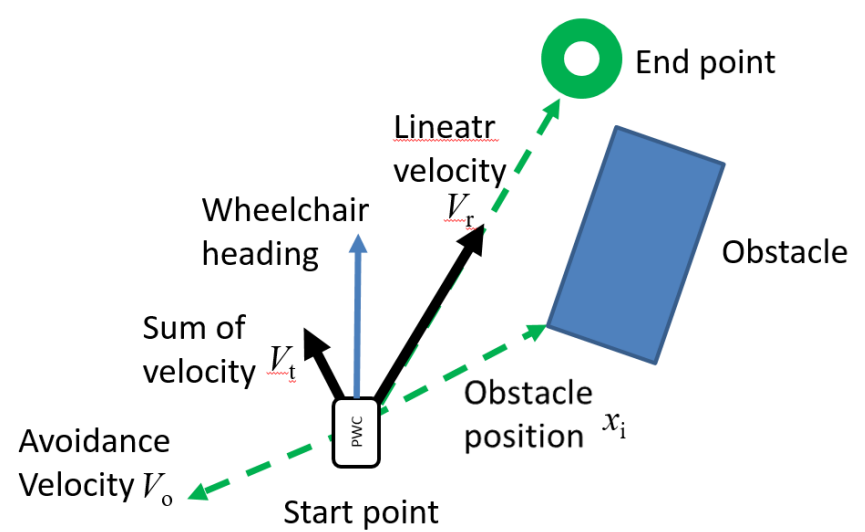

Fig. 3. Obstacle avoider.

There may be more than one obstacle. The sensor system will often only detect the closest object but if more than one object is detected then the obstacle-avoider sums multiple obstacle position vectors to calculate the outputs. To move towards a goal position and prevent collisions, the obstacle resultant velocity, $\boldsymbol{V} t$, is expressed as:

$$
\boldsymbol{V}_{t}=\boldsymbol{V}_{o}+\boldsymbol{V}_{r}
$$

where $\boldsymbol{V}_{r}$ is the original linear velocity from the goal seeking behaviour, $\boldsymbol{V}_{o}$ is the generated avoidance velocity and the resultant velocity, $\boldsymbol{V}_{t}$, is defined in the $\mathrm{X}-\mathrm{Y}$ plane. The command $\boldsymbol{V}_{t}$ is calculated to avoid obstacles without changing the heading of the wheelchair by very much.

\section{SHARED-CONTROLLER}

A joystick provided an interface for control of the wheelchair. Shared-control improved driving by combining sensor system commands and wheelchair driver autonomy.

The skills of the disabled driver can allow them to control the wheelchair safely but the input from the sensor system may be more repeatable and accurate and can compensate for a lack of human driver awareness and ability or misunderstanding of local situations. This study combines the advantages of human driving skills and autonomy, with system intervention when required.

When the sensor system operated on a wheelchair in a complex and varying environment, it provided better decisionmaking than for some disabled drivers. The proposed control architecture for shared-control is illustrated in Fig. 4.

The shared control method is an extension of work in [9] and [35]. The method allows safe and convenient operation of a powered wheelchair.

The wheelchair is usually directed to move in a desired heading using a joystick. The architecture described in this paper combines the information from the joystick to preserve driver autonomy and shares it with input from a sensor system. A user controls the wheelchair's motion through the joystick and can usually see the environment and the wheelchair's location.

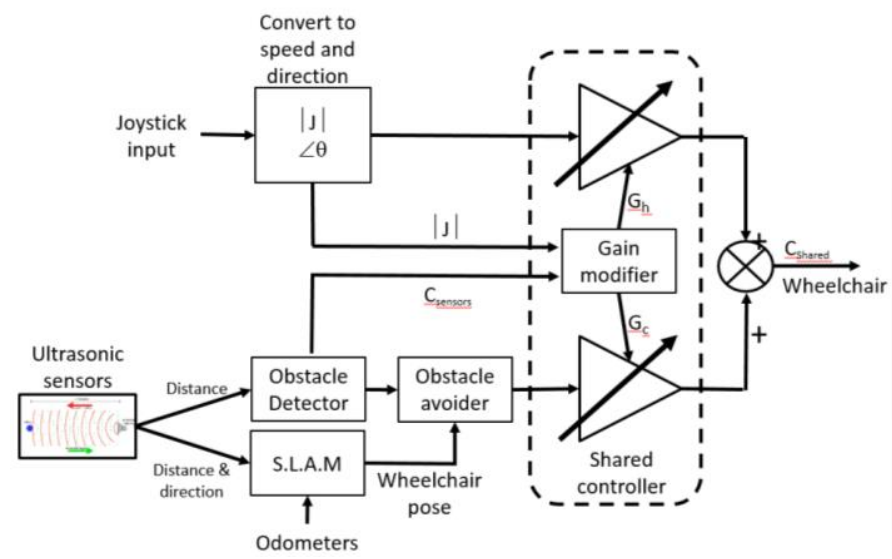

Fig. 4. Shared control.

The wheelchair sensor system handled obstacle avoidance and the obstacle avoider system ensured the wheelchair's safety when it moved.

The motion commands to the wheelchair motors were generated by both the driver and the sensor system. In a situation where there were fewer obstacles or the obstacle(s) were far away, the driver did not need to be assisted by the wheelchair sensor systems. Giving the driver a higher authority ensured improved performance. However in an environment with many obstacles or obstacles close to the wheelchair, the system can reduce or inhibit the joystick commands from the driver in order to prevent any possible collision.

The combined-control gains of the driver and the obstacle avoider are dynamically distributed during movement. As shown in Fig. 4, the resultant control command, $C_{\text {shared }}$ is:

$$
C_{\text {shared }}=G_{h}|\jmath|+G_{c} \boldsymbol{u}_{c} \quad G_{h}, C_{\text {sensors }} \in[0,1]
$$

where $|\mathrm{\jmath}|$ is the motion command from the joystick and $C_{\text {sensors }}$ is the range from the obstacle detector. The output, $C_{\text {shared, }}$ is a summation of the weighted command from the driver. That is, it is multiplied by $G_{h}$, a human-weighted gain, and the weighted command from the autonomous controller output multiplied by $G_{\mathrm{w}}$, a weighted gain,. These gains were established by a self-reliance-factor. The system treats the self-reliance factor of the driver in controlling the wheelchair so as to determine the driver-weighted gain in (13).

\section{A. Self-reliance Factor for avoidance}

The confidence of a driver in controlling a wheelchair was estimated to establish the ranking of the driver's commands. The Self-reliance Factor consisted of three parts. The avoidance-factor was initially set at a value that represented the ability of a driver to cope with potential collisions. It was assumed that drivers had a lower Self-reliance Factor when a wheelchair moved closer to an obstacle or obstruction. $E_{a v o i d}$, the avoidance-factor, was

$$
E_{a v o i d}=\left|x_{0}\right| / D_{\text {Safe }}
$$


where $x_{o}$ was the smallest distance between the wheelchair and any obstacles, obtained from the ultrasonic sensors, and $D_{\text {Safe }}$ was a constant that represented a safe and cautious range. If the powered wheelchair was further away from an obstacle than the constant, $\mathrm{D}_{\text {Safe, }}$, then the driver was assumed to have a greater confidence in driving their wheelchair. If a wheelchair was at a distance less than $D_{\text {Safe }}$ from an obstacle, the user's confidence gradually decreased.

For $x_{0}$ greater than $\mathrm{D}_{\text {Safe }}$, then $\mathrm{E}_{\text {avoid }}$ was saturated as 1 .

\section{B. Self-reliance Factor for safety}

The Self-reliance Factor for safety denoted the ability of a driver to operate the wheelchair safely. If the wheelchair was operated at lower speeds, the driver was assumed to be more confident to ensure safety and stability. A Self-reliance Factor for safety $E_{\text {safety }}$ was defined as:

$$
E_{\text {safety }}=\left\{1-\left(\left|V_{\mathrm{h}}\right| /\left|V_{\mathrm{TH}}\right|\right)\right\}^{q} \text {, for } q<1 \text {. }
$$

where $V_{h}$ was the linear velocity of the user's command, from the joystick. A threshold, $V_{T H}$, was defined as the greatest linear velocity that a user was allowed to drive the wheelchair. In order to ensure that a greater Self-reliance Factor was assigned at lower speeds, this was estimated using an exponent, $\mathrm{q}$ (where $\mathrm{q}<1$ ). $\mathrm{q}$ was determined using observation and experiment. This meant that at slower speeds, a higher Self-reliance Factor could be estimated.

\section{Self-reliance Factor for assistance}

Time and tiredness were often important. If a disabled driver controlled a powered wheelchair continuously, the wheelchair driver was more likely to become tired so that the driver may then have a lower Self-reliance Factor. At the beginning of the day, a driver was more likely to be awake and alert. The disabled driver's control of the joystick during the entire day was considered. The estimated engagement time, $E_{t}$, was defined as

$$
\begin{aligned}
& E_{t}=E_{t-1}+\left(1 / T_{c}\right), \text { if driver had rested } \\
& E_{t}=E_{t-1}-\left(1 / T_{c}\right), \text { if driver was tired }
\end{aligned}
$$

where $T_{c}$ was the adaptive time for a disabled driver in driving the wheelchair. If the user actively controlled the wheelchair then the estimation gradually decreased to zero. If the driver relinquished control and rested, then the estimation increased to one.

\section{Final Self-reliance Factor}

The final Self-reliance Factor and control gains, $\mathrm{G}_{h}$ and $\mathrm{G}_{c}$, were obtained:

$$
\text { ConFact }=E_{t} \times \max \left(E_{\text {avoid }}, E_{\text {safety }}\right)
$$

$\mathrm{G}_{h}=$ ConFact

$$
\mathrm{G}_{c}=1-\text { ConFact }
$$

Self-reliance factor estimations for avoidance and safety were used with the maximum function to give the higher estimated value. When both avoidance and safety self-reliance factors had high values, a driver may drive their wheelchair smoothly. When the estimates for avoidance had a high value, the wheelchair was far from obstacles and the driver was free to control their wheelchair. When the estimates for safety had a high value, the wheelchair was restricted to a relatively low velocity so that the user was safe. This method was useful in avoiding obstacles or approaching an object (for example a wall to turn on a switch) without tiring and tedious manual correction.

\section{EXPERIMENTAL RESULTS}

Several experiments were performed to validate the proposed shared control method.

\section{A. Simulation}

This experimentation and testing validated the design for wheelchair shared-control. The system was simulated on a desk top computer. The joystick input was set to point towards a destination at $3 / 4$ speed.

A typical trajectory for the experiment is shown in Fig. 5.

Figure 5 shows the wheelchair's location and heading at several instants. The powered wheelchair is shown in the figure as:

PWC

At the start, the simulated wheelchair was facing to the right.

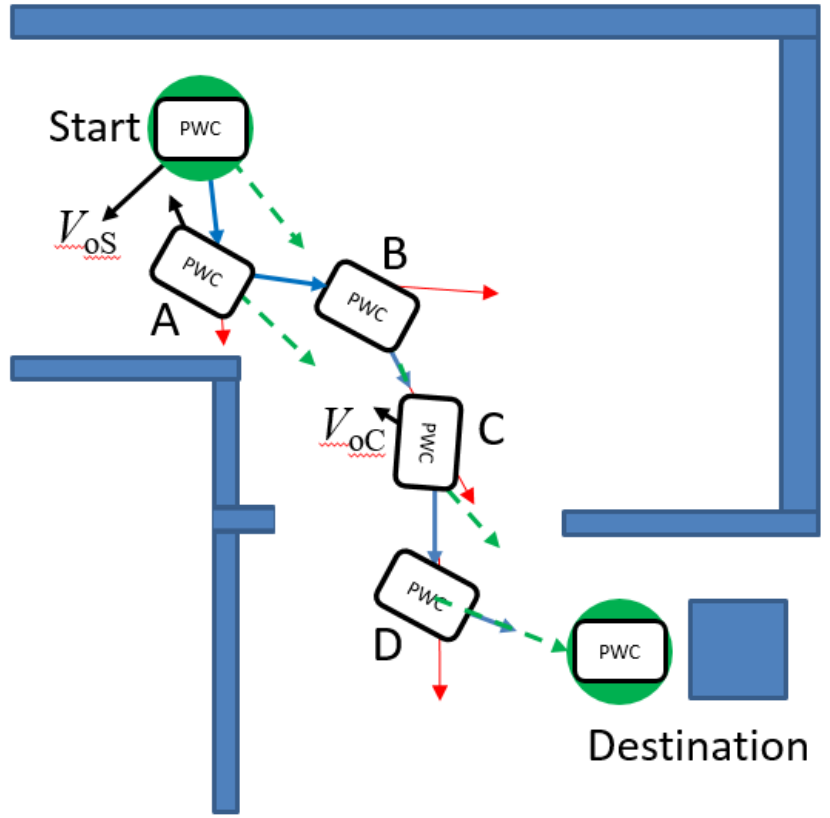

Fig. 5. Simulation experimentation and testing.

The dotted line shows the direction to the destination and therefor the joystick input. The left hand ultrasonic sensor 
detected the wall above the wheelchair and produced $V_{\mathrm{os}}$ to guide the wheelchair away from the wall. The other solid line shows the direction of movement from the Start to Point A. As the wheelchair moved along the path towards Point A, the right hand ultrasonic sensor detected the wall below the wheelchair and generated an avoidance velocity so that the wheelchair turned left to avoid the wall. Once into free space $V_{\mathrm{o}}$, reduced to zero and the wheelchair turned and moved towards the destination under the guidance of the joystick input. At Point $\mathrm{C}$ the left hand sensor had detected the doorway and $V_{\mathrm{oC}}$ was growing so that the wheelchair steered slightly right to avoid the edge of the doorway.

Once through the doorway at Point $\mathrm{D}$, the wheelchair was in free space again and safely turned and moved to the destination.

The simulated wheelchair passed through the environment without colliding with anything and approached the destination safely.

Self-reliance Factor values from the estimators described in section III were recorded at the positions in the figure and were:

$\begin{array}{ll}\text { Start } & 0.4 \\ \text { Point A } & 0.6 \\ \text { Point B } & 1.0 \\ \text { Point C } & 0.7 \\ \text { Point D } & 1.0\end{array}$

The estimation of the Self-reliance Factor value for obstacleavoidance was important when obstacles were detected (the walls), so the driver self-reliance factor decreased when they were detected. The wheelchair slowed down to prevent collision. Because the driver's authority was considered, the sensor system partly controlled the wheelchair until it was in open space. The shared-controller allowed the wheelchair to move away from obstacles while obeying the command from the joystick to move to the destination.

The motion command for the simulated wheelchair was a combination of the simulated input from the joystick and the input from the sensor system, given by (13). These control gains for both the driver and the sensor system resulted in the steering and speed control commands sent to the simulated wheelchair. The simulated wheelchair was guided safely and did not collide with anything.

\section{B. Testing the shared-controller on a wheelchair}

This was to validate the wheelchair shared-control system and to check that the system was safe to use. Volunteer drivers and members of the research staff at Portsmouth drove the wheelchair through doorways, and avoided obstacles to approach a large cardboard box. The drivers controlled the wheelchair using a joystick.

Paths and trajectories were recorded by the odometry and a visual tracker. A typical path is shown in Fig. 6. The location of the wheelchair and the heading are shown for some sample instants. The design of the wheelchair allowed it to turn easily (spinning on it's own axis if required) and then to drive in any direction and to keep a defined heading.

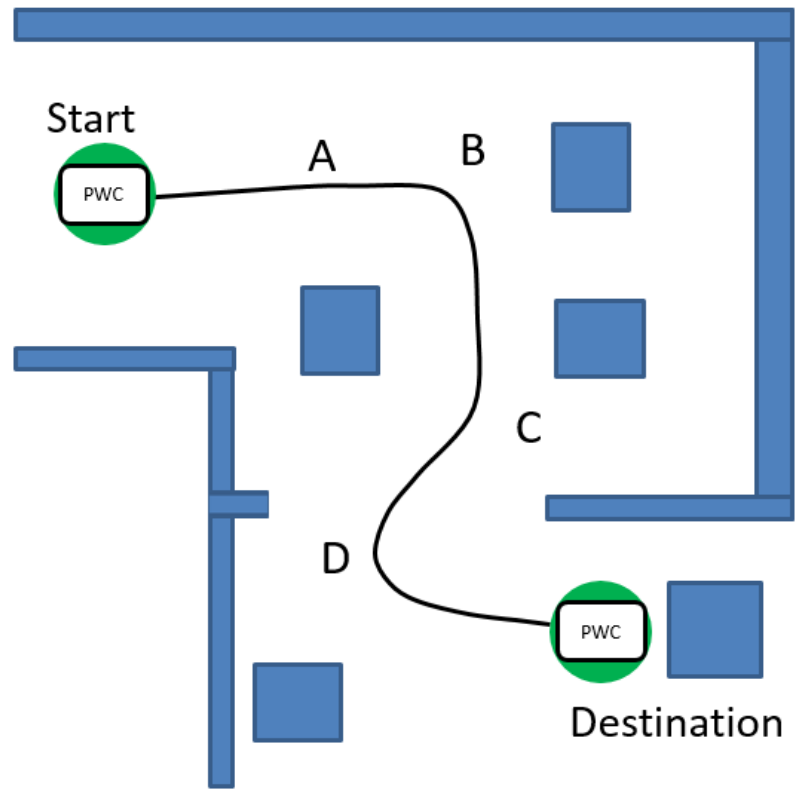

Fig. 6. Recorded trajectory of wheelchair experiment.

That allowed drivers to focus on steering direction and not on avoiding obstacles.

The ultrasonic sensor system assisted the driver in the control of their desired motion via shared-control and compensated for moments when there was a lack of control from the driver. The wheelchair passed through the environment without colliding with anything and safely approached the large box at the destination.

The recorded Self-reliance Factor values for the experiment at the positions in Fig.6. were:

$\begin{array}{ll}\text { Start } & 0.0 \\ \text { Point A } & 0.05 \\ \text { Point B } & 0.64 \\ \text { Point C } & 0.73 \\ \text { Point D } & 0.69\end{array}$

The wheelchair's motion command was a combination of the input from the driver's joystick and the input from the sensor system, given by (13). The control gains for both the driver and the sensor system resulted in the speed (and therefor also the direction) commands sent to the wheelchair motors.

Driver self-reliance factor decreased when obstacleavoidance was required and the wheelchair was guided safely and did not collide with anything.

The final distance between the wheelchair and the large box was only $5 \mathrm{~cm}$.

If the user slowed down during the test then it was safer to move and hence the driver maintained high authority. The wheelchair slowed down to prevent collision at the destination. Because the driver's authority was considered, the sensor system remotely controlled the wheelchair until it was very close to the box at the destination. The sharedcontrol allowed the wheelchair to travel around (or away from) obstacles while obeying a driver's command to drive towards the box at the destination. 


\section{Comparison of driving with and without the controller}

Driving with and without the controller was compared for a small group of testers and recognized procedures were followed for human subject inclusion. The purpose was to validate that shared-control was useful during wheelchair driving. The experimental environment setup was the same as that for the previous shared-control experiment. Each experiment contained two parts: driving without the system and then again with the system engaged.

The results from three subjects are shown: one female aged 15 (Driver A), one male aged 17 (Driver B) and one male aged 18 (Driver C). Each subject conducted two trials, one with the system engaged and one without. The interval between consecutive trials was more than 12 hours to allow plenty of time for rest and recovery.

The time taken for each trial is shown in Fig. 7.

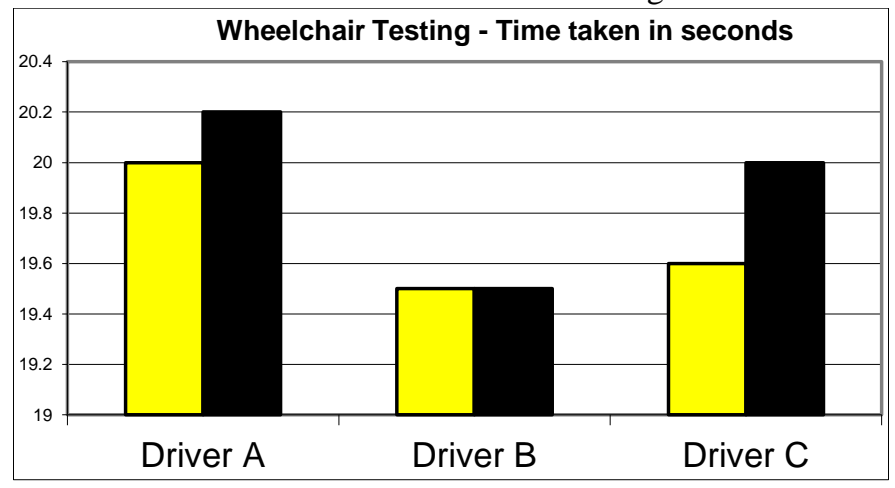

Fig. 7. Time taken (s) to complete the course form Start to Destination.

The times taken with the sensor system engaged are shown on the left. Times taken without the sensor system engaged are shown on the right. Results showed that the route was generally completed more quickly with the sensor system engaged. Execution time for shared-control was generally less than that normal driving.

The number of collisions recorded for each trial is shown in Fig. 8.

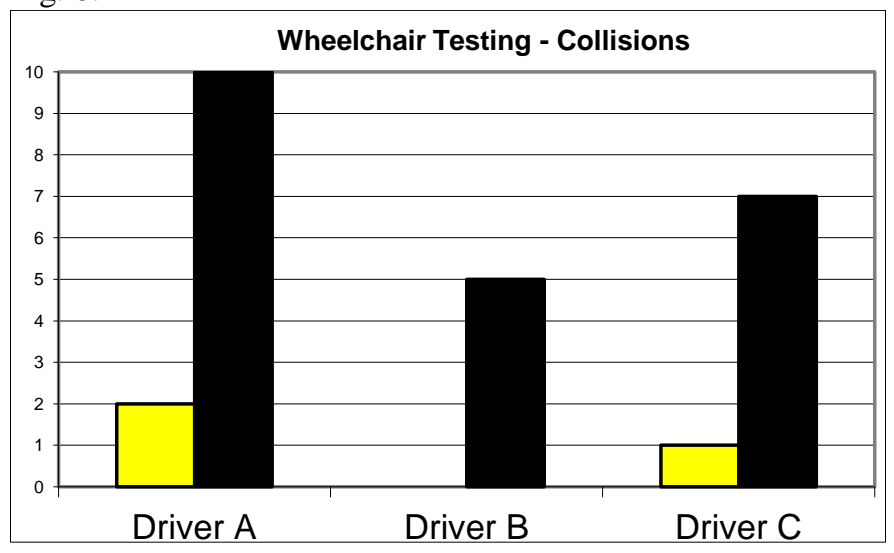

Fig. 8. Collisions recorded for each driver.

The number of collisions was recorded for each driver. The number of collisions when the sensor system was engaged are shown on the left. The number of collisions without the sensor system engaged are shown on the right. Results showed that the route was completed with fewer collisions (if any) when the sensor system was engaged.

Execution time and safety for shared-control was generally less than that normal driving.

The main reason for these improvements was that the driver no longer needed to closely monitor wheelchair motion as it moved close to obstacles. If the operator used more effort to avoid the obstacles, more time was required to ensure safety. However, with shared-control, the drivers were safe and they took less time to negotiate obstacles with assistance from the sensors.

\section{DISCUSSION AND CONCLUSIONS}

A new shared-control architecture has been designed and implemented for a powered wheelchair. The driver is in control of the wheelchair unless a sensor system needs to provide some assistance, for example to avoid an obstacle or to pass through a doorway.

The work does not deal with obstacles above and below the volume of the ultrasonic sensors.

The amount of input provided by the driver and the amount provided by the sensor system were determined by estimating some human self-reliance factors. These were determined dynamically using sensory information, for example how fast is the wheelchair going, how close is the wheelchair to an obstacle and how long has the wheelchair been operating.

Wheelchair drivers controlled the wheelchair more easily if there was assistance from the wheelchair's sensor during execution. Experimental results demonstrated that sharedcontrol was safe and helpful for avoiding obstacles and approaching a destination efficiently.

There is an optimal mixture of autonomous versus human control for different wheelchair drivers and in different circumstances, for example whether the driver is tired or not. This optimum changes with the skill and experience of the human operator.

\section{FUTURE WORK}

The static sensor array has started to limit the ongoing work as the position of obstacles is not accurate enough to provide data for improved algorithms. Adding more sensors was considered but instead, some scanning collision avoidance detectors have been developed by Langner at the Chailey Heritage Foundation. Those scanning ultrasonic devices will be used in future research work as they are smaller and less obtrusive, they cover a wider area and position and range can be accurately detected. The wheelchair sensor systems described in this paper are being dismantled and the scanning devices are being tested on a new set of powered wheelchairs at the University of Portsmouth at the time of writing. Some early results from that testing can be seen at:

https://www.facebook.com/PortsmouthUniversityMobility

The odometers that helped to build the simple map in the database proved to be inaccurate over longer distances $(>50 \mathrm{~m})$, and they proved to be largely unnecessary. Other AI systems are being considered and tested at Portsmouth [43-52] but they are all tending to be more complicated and more expensive. An aim in any work involving disability assistance is to keep the work simple and cheap if possible and it is 
expected that the scanning sensors and a simple microcontroller will be all that are required in the future.

Other research is evaluating the effect of this sensor / control technology on user acceptance.

Wheelchair users are already beginning to rely on some sensor systems. The system be can be customized and adjusted for individual users to meet individual needs (for example for varying levels of cognitive and / or physical ability by adjusting the Self-reliance Factor and gains.

The technology is already being tied in to other systems developed at Portsmouth [3-6, 13-18].

\section{REFERENCES}

[1] I.M. Mitchell, P. Viswanathan, B. Adhikari, E. Rothfels, and A.K. Mackworth. "Shared Control Policies for Safe Wheelchair Navigation of Elderly Adults with Cognitive and Mobility Impairments: Designing a Wizard of Oz Study", Proc' of the American Control Conf, 2014, pp 4087-4094.

[2] L. Fehr, W.E. Langbein, and S.B. Skaar. "Adequacy of power wheelchair control interfaces for persons with severe disabilities: A clinical survey", Journal of Rehabilitation Research and development, vol. 37, no. 3 pp. 353360, 2000.

[3] M.J. Goodwin, D.A. Sanders G.A. Poland et al. "Navigational assistance for disabled wheelchair-users", Proc' Euromicro Conf 95, vol. 43, pp. 73-79, 1997.

[4] I. Stott and D.A. Sanders, "The use of virtual reality to train powered wheelchair users and test new wheelchair systems", International Journal of Rehabilitation Research: vol. 23, no. 4, pp. 321-326, 2000.

[5] D. A. Sanders, G. E. Tewkesbury, D. Ndzi, et al. "Improving automatic robotic welding in shipbuilding through the introduction of a corner-finding algorithm to help recognise shipbuilding parts", Journal of Marine Science and Technology vol. 17, no. 2 pp. 231-238, 2012.

[6] D. Sanders, "Comparing speed to complete progressively more difficult mobile robot paths between human tele-operators and humans with sensorsystems to assist", Assembly Automation, vol. 29, no. 3, pp. 230-248. 2009.

[7] A. Ruiz-Serrano, R. Posada-Gomez, A. M. Sibaja, G. A. Rodriguez, B. E. Gonzalez-Sanchez, O. O. Sandoval-Gonzalez, Edited by: M.L Rivera, "Development of a dual control system applied to a smart wheelchair, using magnetic and speech control", Proc $3^{\text {rd }}$ IberoAmerican Congf on Electronics Emgineering and computer science, CIIECC 2013, Book Series: Procedia Technology, vol. 7, pp. 158-165. 2013.

[8] R. Zhang, Y. Li, Y. Yan, H. Zhang, S. Wu, T. Yu, and Z. Gu, "Control of a Wheelchair in an Indoor Environment Based on a Brain-Computer Interface and Automated Navigation", IEEE Transactions on neural systems and rehabilitation engineering: a publication of the IEEE Engineering in Medicine and Biology Society vol. 24, no. 1, pp. 128-39, 2016.

[9] D. A. Sanders, N. Bausch, H. Liu, et al., "Improving Steering of a Powered Wheelchair Using an Expert System to Interpret Hand Tremor", Proc Intelligent Robotics and Applications (ICIRA 15), Pt I, vol 9245, pp. 460-471, 2015.

[10] L. S. Schmalfu, R. Rupp, M. R. Tuga, A. Kogut, M. Hewitt, J. Meincke, F. Klinker, W. Duttenhoefer, U. Eck, and R. Mikut, "Steer by ear: Myoelectric auricular control of powered wheelchairs for individuals with spinal cord injury", Restorative neurology and neuroscience, vol. 34, no. 1, pp. 79-95, 2015.

[11] A. Phinyomark, P. Phukpattaranont and C. Limsakul, "A Review of Control Methods for Electric Power Wheelchairs Based on Electromyography Signals with Special Emphasis on Pattern Recognition", IETE Technical Review, vol. 28, no. 4, pp. 316-326, 2011.

[12] R. Maskeliunas and R. Simutis. "Multimodal Wheelchair Control for the Paralyzed People" Electronics and Electrical Engineering. - Kaunas: Technologija, 2011, vol. 5, no. 111, pp. 81-84.

[13] D. A. Sanders, I. J. Stott, J. Graham-Jones, et al., "Expert system to interpret hand tremor and provide joystick position signals for powered wheelchairs with ultrasonic sensor systems", Industrial Robot-an International Journal, vol. 38, no. 6, pp. 585-598, 2011.

[14] D. A. Sanders, M. Langner and G. E. Tewkesbury, "Improving wheelchair-driving using a sensor system to control wheelchair-veer and variable-switches as an alternative to digital-switches or joysticks", Industrial Robot-an International Journal, vol. 37, no. 2, pp. 157-167, 2010.
[15] D. A. Sanders, I. J. Stott, D. C. Robinson, et al., "Analysis of successes and failures with a tele-operated mobile robot in various modes of operation", Robotica, vol. 30, pp. 973-988, 2012.

[16] I. J. Stott and D. A. Sanders, "New powered wheelchair systems for the rehabilitation of some severely disabled users", Int Jrnl of Rehab Research, vol. 23, no. 3, pp. 149-153, 2000.

[17] D. A. Sanders, J. Graham-Jones, and A. Gegov, "Improving ability of tele-operators to complete progressively more difficult mobile robot paths using simple expert systems and ultrasonic sensors", Industrial Robot-an International Journal, vol. 37, no. 5, pp. 431-440, 2010.

[18] D. A. Sanders and I. J. Stott, "A new prototype intelligent mobility system to assist powered wheelchair users", Industrial Robot, vol. 26,no. 6, pp. 466-475, 1999.

[19] K. Kuniaki, I. Tatsuya, F. Teruo, N. Takashi, A. Asama and E. Isao, "Teleoperation of Autonomous Mobile Robot under Limited Feedback Information," in Proc. of International Conf on Field and Service Robotics, London, United Kingdom, 1998, pp 146-151.

[20] D. G. Macharet and D. A. Florencio, "A Collaborative Control System for Telepresence Robots," in Proc. of IEEE/RSJ International Conf on Intelligent Robots and Systems, Algarve, Portugal, 2012, pp. 5105-5111.

[21] S. Hayati and S. T. Venkataraman, "Design and Implementation of a Robot Control System with Traded and Shared Control Capability," in Proc. of IEEE International Conf on Robotics and Automation, Scottsdal, AZ, USA, 1989, pp. 1310-1315.

[22] A. David M. M. Abbink, and E. R. Boer, "Haptic Shared Control: Smoothly Shifting Control Authority?," Cognition, Technology \& Work, Springer-Verlag, vol. 14, no. 1, pp. 19-28, 2012.

[23] Makoto Itoh, Toshiyuki Inagakia and Hiroto Tanaka, "Haptic Steering Direction Guidance for Pedestrian-Vehicle Collision Avoidance," in Proc. of IEEE Int Conf on System, Man, and Cybernetics, Seoul, Korea, 2012, pp. 3309-3314.

[24] T. Carlson and Y. Demiris, "Increasing Robotic Wheelchair Safety With Collaborative Control: Evidence from Secondary Task Experiments," in Proc. of IEEE International Conf on Robotics and Automation, Anchorage, Alaska, USA, 2010, pp. 5582-5587.

[25] A. R. Satti, D. Coyle and G. Prasad, "Self-paced Brain-controlled Wheelchair Methodology with Shared and Automated Assistive Control," in Proc. of IEEE Symposium on Computational Intelligence, Cognitive Algorithms, Mind, and Brain, Paris, France, 2011, pp. 1-8.

[26] A. Franchi, C. Secchi, M. Ryll, H. H. Bulthoff and P. R. Giordano, "Shared Control : Balancing Autonomy and Human Assistance with a Group of Quadrotor UAVs," IEEE Robotics \& Automation Magazine, vol.19, no.3, pp. 57-68, 2012

[27] L. Tonin, R. Leeb, M. Tavella, S. Perdikis and J. del R. Millan, "The Role of Shared-Control in BCI-based Telepresence," in Proc. of IEEE International Conf on Systems, Man, and Cybernetics, Istanbul, Turkey, 2010, pp. 1462 - 1466.

[28] H. K. Kim, S. J. Biggs, D. W. Schloerb, J. M. Carmena, M. A. Lebedev, M. A. L. Nicolelis and M. A. Srinivasan, "Continuous Shared Control for Stabilizing Reaching and Grasping With Brain-Machine Interfaces," IEEE Transactions on Biomedical Engineering, vol. 53, no. 6, pp. 11641173. 2006

[29] D.A. Sanders, S.D. Urwin-Wright, G.E. Tewkesbury, GE; et al. "Pointer device for thin-film transistor and cathode ray tube computer screens", Electronics Letters, vol. 41, no. 16, 2005, pp. 894-896.

[30] D.A. Sanders, "Analysis of the effects of time delays on the teleoperation of a mobile robot in various modes of operation", Ind Robot vol. 36, no. 6, 2009, pp. 570-584.

[31] D.A. Sanders, "Controlling the direction of "walkie" type forklifts and pallet jacks on sloping ground", Assembly Automation, vol. 28, no. 4, 2008, pp. 317-324.

[32] D.A. Sanders and J Bergasa-Suso, "Inferring Learning Style From the Way Students Interact With a Computer User Interface and the WWW", IEEE Transactions on Education vol. 53, no. 4, 2010, pp. 613-620.

[33] D.A. Sanders and G.E Tewkesbury, "A pointer device for TFT display screens that determines position by detecting colours on the display using a colour sensor and an Artificial Neural Network", Displays, vol. 30, no. 2, 2009, pp. 84-96.

[34] D.A. Sanders and A Baldwin, "X-by-wire technology", Total Vehicle Technology Conference, ISBN:1-86058-324-5, pp. 3-12. 2001.

[35] D.A. Sanders, "Comparing ability to complete simple tele-operated rescue or maintenance mobile-robot tasks with and without a sensor system", Sensor Review vol. 30, no. 1, 2010, pp. 40-50.

[36] T. Carlson and Y. Demiris, "Collaborative Control for a Robotic Wheelchair: Evaluation of Performance, Attention, and Workload," IEEE 
Trans. on Systems, Man, and Cybernetics-Part B: Cybernetics, vol. 42, no. 3, 2012, pp. 876-888.

[37] J. Kim, D. Chang, H. Ladjal, D. Folio, A. Ferreira and J. Kim, "Evaluation of Telerobotic Shared Control for Efficient Manipulation of Single-Cells in Microinjection," in Proc. of IEEE Int Conf on Robotics and Automation, Shanghai, China, 2011, pp. 3382-3387.

[38] Q. Li, W. Chen and J. Wang, "Dynamic Shared Control for HumanWheelchair Cooperation," in Proc of IEEE International Conf on Robotics and Automation, Shanghai, China, 2011, pp. 4278-4283.

[39] T. Carlson, R. Leeb, R. Chavarriage and J. del R. Millan, "Online Modulation of the Level of Assistance in Shared Control System," in Proc of IEEE Int Conf on System, Man, and Cybernetics, Seoul, Korea, 2012, pp. 3321-3326.

[40] S. Y. Jiang and K-T. Song, "Differential Flatness-based Motion Control of a Steer-and-Drive Omnidirectional Mobile Robot," in Proc. of IEEE Int Conf on Mechatronics and Automation, Kagawa, Japan, 2013, pp. 1167-1172. [41] D.A. Sanders "Helping disabled users to drive powered wheelchairs using ultrasonic sensors and a rule-based artificial neural network", submitted to IEEE Transactions on Human Machine Systems, In Press.

[42] K. T. Song, S. Y. Jiang, M. H. Lin, C. H. Wu, Y. F. Chiu, W. C. Lin S. Y. Wu and C. Y. Wu, "Design and Experiment of a Human-Touch Robot," in Proc. of CACS International Automatic Control Conf, Kaohsiung, Taiwan, 2014, pp. 275-280.

[43] D.A. Sanders, "Recognizing shipbuilding parts using artificial neural networks and Fourier descriptors", Proceedings of the Institution of Mechanical Engineers Part B-Journal of Engineering Manufacture vol. 223 no. 3, 2009, pp. 337-342.

[44] D.A. Sanders, B.P. Haynes and G.E. Tewkesbury, "The addition of neural networks to the inner feedback path in order to improve on the use of pre-trained feed forward estimators", Mathematics and Computers in Simulation vol. 41, no. 5-6, 1996, pp. 461-472.

[45] D.A. Sanders and A. Gegov, "AI tools for use in assembly automation and some examples of recent applications", Assembly Automation vol. 33, no. 2, 2013, pp. 184-194.

[46] D.A. Sanders, Y.C. Tan, I. Rogers et al, "An expert system for automatic design-for-assembly", Assembly Automation vol. 29, no. 4, 2009, pp. 378-388. [47] D.A. Sanders and A.D. Hudson, "A specific blackboard expert system to simulate and automate the design of high recirculation airlift reactors", Mathematics and computers in simulation, vol. 53, no. 1-2, 2000, pp. 41-65.

[48] D.A. Sanders, "Progress in machine intelligence", Ind Robot, vol. 35, no. 6, 2008, pp. 485-487.

[49] D.A. Sanders, "Introducing AI into MEMS can lead us to brain-computer interfaces and super-human intelligence", Assembly Automation, vol. 29, no. 4, 2009, pp. 309-312.

[50] A. Gegov, F. Arabikhan and D.A. Sanders, "Rule base simplification in fuzzy systems by aggregation of inconsistent rules", Journal of Intelligent \& Fuzzy Systems, vol. 28, no. 3, 2015, pp. 1331-1343.

[51] D.A. Sanders, G.E. Tewkesbury, I.J. Stott et al, "Simple expert systems to improve an ultrasonic sensor-system for a tele-operated mobile-robot", Sensor Review vol. 31, no. 3, 2011, pp. 246-260.

[52] D.A. Sanders, N.Bausch, et al, "Improving Steering of a Powered Wheelchair Using an Expert System to Interpret Hand Tremor", Intelligent Robotics and Applications (ICIRA 2015), Pt I vol. 9245, 2015, pp. 460-471.

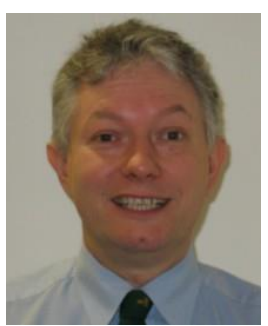

David A. Sanders TD VR CEng was born in London, UK in 1958. He received two first degrees, a $\mathrm{BA}$ in Social Science and Mathematics in 1985 (OU), and a BSc in Engineering in 1984 (Portsmouth, UK). He went on to post graduate study and was awarded a DMS in Management Studies and then a MBA in Business Administration and Change Management in 2001 (OU), finally he completed a $\mathrm{PhD}$ in Automatic Path Planning with constraints in 1990 (CNAA, UK).

Since 2002 he has been a consultant engineer and the READER in Systems \& Knowledge Engineering in the School of Engineering at the University of Portsmouth, except for breaks on mobilized military service as a Colonel in the UK Army and working for the Royal Academy of Engineering. $\mathrm{He}$ is the author of three books, more than 250 articles, and several patents and inventions. Current and previous research interests include assistive technologies, sensor integration, robotics, tele-operation, powered wheelchairs and obstacle avoidance.

Dr. Sanders is a Fellow IET, Fellow IMechE, and Fellow HEA. 